University of Nebraska - Lincoln

DigitalCommons@University of Nebraska - Lincoln

$5-2006$

\title{
Modeling and Representation of Geometric Tolerances Information in Integrated Measurement Processes
}

Xiaoping Zhao

T. M. Kethara Pasupathy

Robert G. Wilhelm

Follow this and additional works at: https://digitalcommons.unl.edu/mechengfacpub

Part of the Mechanics of Materials Commons, Nanoscience and Nanotechnology Commons, Other Engineering Science and Materials Commons, and the Other Mechanical Engineering Commons

This Article is brought to you for free and open access by the Mechanical \& Materials Engineering, Department of at DigitalCommons@University of Nebraska - Lincoln. It has been accepted for inclusion in Mechanical \& Materials Engineering Faculty Publications by an authorized administrator of DigitalCommons@University of Nebraska Lincoln. 
Published in Computers in Industry 57:4 (May 2006), pp. 319-330; doi: 10.1016/j.compind.2005.09.004 Copyright (c) 2005 Elsevier B.V. Used by permission.

Submitted April 16, 2004; accepted September 12, 2005; published online November 23, 2005.

\title{
Modeling and Representation of Geometric Tolerances Information in Integrated Measurement Processes
}

\author{
Xiaoping Zhao, T. M. Kethara Pasupathy, and Robert G. Wilhelm
}

Center for Precision Metrology and Manufacturing, Department of Mechanical Engineering, University of North Carolina at Charlotte, Charlotte, North Carolina, USA

Corresponding author - Xiaoping Zhao, Center for Precision Metrology and Manufacturing, Department of Mechanical Engineering, University of North Carolina at Charlotte, 9201 University City Blvd., Charlotte, NC 28223, USA, telephone +1 704687 4689, fax +1 704687 3246, email xzhao@uncc.edu

\begin{abstract}
Modeling and representation of geometric tolerances information across an enterprise is viable due to the advances in Internet technologies and increasing integration requirements from industry. In Integrated Measurement Processes (IMP), geometric tolerances data model must support different models from several well-defined standards: including ASME Y14.5M-1994, STEP, DMIS, and others. In this paper, we propose a layered conformance level geometric tolerances representation model. This model uses the widely applied ASME Y14.5M-1994 as its foundation layer by abstracting most information from this standard. The additional geometric tolerances information defined by DMIS and STEP is incorporated into this model to form corresponding conformance layers that support IMP. Thus, different application domains in an enterprise can use this data model to exchange product information. This model is further transformed with XML Schema that can be used to generate $\mathrm{XML}$ instance file to satisfy geometric tolerances representation requirements in IMP.
\end{abstract}

Keywords: geometric tolerances modeling and representation, integrated measurement processes, ASME Y14.5M-1994, STEP, DMIS 


\section{Introduction}

Tolerancing is widely used in industry to define the allowable variation of parts from their ideal shape. The tolerance information plays a critical role in many steps of the product life cycle, such as design, manufacturing, inspection, assembly, and so on with different emphasis. Among many research efforts related with tolerance, tolerance modeling and representation is one of important and active research fields [1]. Modeling and representing tolerance information with a unified, unambiguous, computer and human understandable data model is essential in today's industry when we are in the age of Internet and e-business. The ultimate goal is to ensure product quality while reducing product cost.

Due to inevitable errors introduced in manufacturing processes, different measurement processes are used to inspect the part to verify if it is within or outside of specified geometric tolerancing zones. With the continuous diffusion of CAD/CAM technology, Integrated Measurement Processes (IMP) is emerging to integrate the inspection activities within already well-defined CAD/CAM systems to achieve higher efficiency and more data sharing. In this area, Feng identifies the application activity model to summarize some functional requirements and activities of IMP [2]; Zhao et al. suggest some important aspects and applicable technologies in IMP [3]; NIST presents an effort to integrate dimensional measurement machines' controller using I++ interface [4]. Fundamental to the IMP, is the requirement to provide a harmonized data model and represent it appropriately, including geometric tolerances information that can be understood well by computers and users.

Most of the CAD/CAM systems support the widely accepted ISO 10303-STandard for the Exchange of Product model data (STEP) to encapsulate tolerance data. STEP describes geometric tolerances information via EXPRESS using hierarchical structure. A similar data model is the ANSI/CAM-I 104.0-2001 - Dimensional Measuring Interface Standard (DMIS) for measurement processes that describes geometric tolerances information with a flat structure. Though both the systems are well developed, they use different description mechanisms to satisfy the constraints of respective applications. A mechanism to integrate these two tolerance representation formats is essential. This paper presents a methodology for data modeling and representation of geometric tolerances information for IMP.

From system integration viewpoint, instead of point-to-point transformation, a neutral data file format is often presented to efficiently integrate heterogeneous application systems. Thus, the associated transformation cost can be reduced dramatically [5]. In this paper, we present XML (eXtensible Markup Language) as a neutral data file to represent geometric tolerances information due to the many inherent advantages of XML file.

The remainder of this paper is organized as follows. Section 2 reviews related work in this area and illustrates our motivation. Section 3 concisely describes the solution to address tolerance representation requirements in IMP using XML serial technologies. Section 4 presents a layered conformance level data model using a series of tables and EXPRESS-G diagrams. Section 5 illustrates the representation of that layered data model with XML Schema. Section 6 shows the process by which an example part can use our data model to construct a valid XML instance file to be used in IMP. Section 7 concludes this paper. 


\section{Literature review and motivation}

So far, research work in geometric tolerances modeling and representation can be classified into two categories: system-dependent and system-independent methods. In the first category, research focus on modeling and representation of tolerance information within a geometric modeling system. In the second category, research focus on independently modeling and representing tolerance information. Since our work falls into the second category, we review the literature in this category. For the first category, interested readers can refer to Requicha's work in CSG (constructive solid geometry) [6-8] and recent work in CSG-Brep hybrid geometric modelers $[9,10]$.

In the second category, Bjorke proposed the Tolerance Chain to represent the dimensional relations among surface features [11]. Though the Tolerance Chain was intended for dimensional tolerances, it is also applicable to geometric tolerances. Tsai and Cutkosky presented a graph-based representation scheme to represent geometric tolerances information across a part or assembly called a Tolerance Network [12]. Their data model is associated with the decomposition of a hierarchical product model and also uses nodes and arcs as in Bjorke's model.

Also, there are some research efforts that are closely related to the ANSI Y14.5 standard. Guilford and Turner classified several representational primitives for geometric tolerances based on ANSI Y14.5-1982 [13]. Zeid modeled tolerances with an attributed graph structure that represents ANSI Y14.5-1982 tolerance classes [14]. Feng and Eugene represented tolerance information in class diagrams with Unified Modeling Language [15].

Despite all those efforts, the most relevant and detailed prior work to this paper's focus is works from two well-known committees: STEP and DMIS. Currently, STEP is a de facto standard in CAD/CAM field and DMIS is a de facto standard in measurement processes among metrology groups. Since STEP's geometric tolerances information model is based on ISO standards while DMIS's model is based on ASME Y14.5M-1994, it is worthwhile to have a brief review of those two standards and then look into these two committees' work.

\subsection{Related standards}

The ASME Y14.5M-1994 is revised from the previous ANSI Y14.5M-1982 by introducing some new concepts and symbols and adapting to international standards. DMIS 4.0 uses this standard to model and represent geometric tolerances [16]. Geometric tolerances in ISO are mainly illustrated in ISO 1101:1983 [17]. Both standards specify the semantics and symbols to present tolerance information in engineering drawings. However, additional structure and encoding is still required to model and represent these tolerance definitions in computer-interpretable and human-understandable ways.

The main difference between the ASME and ISO standards relates to limits of size. The ASME standard is based on the "envelope principle" (Taylor Principle, Rule \#1) [18] while the ISO standard is based on the Independency Principle [19]. Though two standards have different principles, most of the symbology is identical and ISO standard also allows user to apply the Taylor Principle to the toleranced feature either by reference to ASME Y14.51994 or by using symbol E. Thus, this paper focuses solely on the ASME standard to build geometric tolerances foundation level. 


\subsection{Committee works}

\subsubsection{STEP geometric tolerances information model}

STEP is a set of standards for a computer-interpretable representation and exchange of product data to provide a mechanism capable of describing product data throughout its life cycle while independent of any specific system [20]. STEP consists of different parts including description methods, integrated resources, and application protocols (AP), as well as implementation methods and abstract test suites. Geometric tolerances information model in STEP is based on ISO serial tolerance standards. Such information can be found in AIC 519 (Application Interpreted Constructs) [21] and some APS, such as AP224 [22]. It also uses some entities from Part 47 [23]. Its main focus is to meet the design phase requirements. Besides STEP work, there are some individual authors presented tolerance models, such as Feng and Yang's work [24] and the further refinement work from Tsai et al. [25] by adding mating relationship between parts and datum reference frame. All such information is documented using EXPRESS and EXPRESS-G, which provides an object-oriented information modeling way to describe hierarchical product data $[26,27]$.

STEP AP219 is a new effort to retouch the geometric tolerances information model by referring to other STEP APs and AIC 519. Its scope is to specify information requirements for analyzing the data and reporting the results of dimensional inspections of solid parts or assemblies [28]. Current version of AP219 uses similar geometric tolerances information model as other APs and imports some attributes from another group's work-Dimensional Markup Language group (DML) [29]. DML's focus is to represent dimensional inspection results with XML based on some information from standards and practical considerations. Since AP219 is still under development, we hope our work can be useful for that committee.

\subsubsection{DMIS geometric tolerances information model}

DMIS is a standard created by the Consortium for Advanced Manufacturing International to enable dimensional measurement machines to communicate seamlessly with each other. Each tolerance in DMIS is represented individually using Backus-Naur Form in terms of tolerance nominal and tolerance actual [16]. Tolerance nominal modeling is based on the semantic interpretation of ASME Y14.5M-1994 standard. Tolerance actual modeling is based on tolerance nominal definition plus some unique terms related with inspection procedure, like INTOL/OUTOL, lim, and so on.

\subsection{Motivations}

From the previous literature review, we realize that there is not an existing suitable way to model and represent geometric tolerances in IMP currently. STEP and DMIS address this issue from their respective viewpoint with limited scope and information and are dominant in design and inspection domains individually. Also, we lack a data model to include as much geometric tolerances information as possible from ASME Y14.5M-1994. Such information appears in feature control frame and some notations in the standard as discussed in Section 4.1 and should be included in the data model obviously. Thus, a new data structure is needed to model and represent geometric tolerances information in IMP 
to catch maximum possible information from ASME Y14.5M-1994 and incorporate these two committees' work. Other requirements for such a data structure include:

- It should represent tolerance information in an objected-oriented way to meet different application domains' requirements by taking information from appropriate layer.

- It should be defined in a format to make it computer interpretable and humanunderstandable. The semantic meanings can be traced to relevant standards.

- It should consider the new trends in industry. In such a situation, web-based applications are often used to exchange product data in distributed manufacturing environments [30-32]. Thus, the data format should be web-applicable.

\section{Solution based on XML and XML Schema}

In this section, we first justify the choice of XML-related technologies to represent tolerance information in IMP, and then a solution based on those technologies is proposed with concise illustrations. This solution is then detailed in Sections 4 and 5 .

\subsection{Why XML-related technologies}

The use of the Internet, the World Wide Web, and XML is a de facto requirement in new system integration projects that address application interoperability and data exchange [30-32]. There are two reasons for this. One reason is that the ubiquity and acceptance of the Internet and World Wide Web provide the furthest-reaching and lowest-cost integration platform in the world. Another reason is the advantages of using XML since it is W3C's recommendation, half-structured and presentation-free and so on. Also, XML bindings of EXPRESS schema and corresponding data are standard implementation methods for STEP [33].

For the XML to be more robust, an XML Schema file is needed to define the data structure of XML data. After a user constructs an XML instance file, its validity can be pre-checked with the schema before it is passed to another system.

After comparing several available XML Schemas in the market, XML Schema [34] becomes the choice of our approach to represent tolerance information in IMP. The reasons to choose XML Schema over others are as follows:

- XML Schema itself uses XML syntax to take advantage of XML benefits.

- It supports a rich set of built-in datatypes and allows user to define their own datatypes.

- It supports datatype inheritance, which is suitable for object-oriented modeling.

- It provides some data constraints.

- It supports namespace hooks, thus provides a systematic way to manage schema files by putting different kinds of schema files in different namespaces.

\subsection{Solution}

According to the properties of XML Schema and requirements to represent tolerance in IMP, this paper shows how to represent tolerance information by using XML Schema in 
multiple-layered conformance levels to meet different application domains' requirements. Based on the XML Schema file, users can construct an appropriate XML instance data file to meet requirements. Such an XML instance file can be used in IMP and is suitable for web-based application throughout the Internet.

\section{Layered conformance level data modeling}

In this section, we follow the process of data modeling [24] by first creating data requirements based on ASME Y14.5M-1994 standard, then abstracting those information in tables and documenting them with EXPRESS-G diagram to provide a formal object-oriented and graphical view of geometric tolerances information [27]. Since the development of geometric tolerances information model in DMIS is based on ASME Y14.5M-1994, it is modeled as the second layer. The geometric tolerances representation in IMP is modeled in the third layer since it needs to include information from both DMIS and STEP.

\subsection{Foundation layer}

As mentioned above, the ASME Y14.5M-1994 standard is used as foundation layer. In this standard, 14 types of geometric tolerances are defined. They are form (straightness, flatness, circularity, and cylindricity), profile (profile of a line and profile of a surface), orientation (angularity, perpendicularity, and parallelism), location (position, concentricity, and symmetry), and runout (circular runout and total runout). Each type has an implicit or explicit 2-D or 3-D tolerance zone type. Tolerance zone types in 3-D include parallel planes, square/rectangular, cylinder, spherical, uniform boundary, and conical and concentric cylinders. Tolerance zone types in 2-D include parallel lines, circular, uniform boundary, concentric circles [35].

From this standard, all 14 types of geometric tolerances information are specified via a feature control frame. Figure 1 shows a typical feature control frame and possible symbols that may appear in it [35].

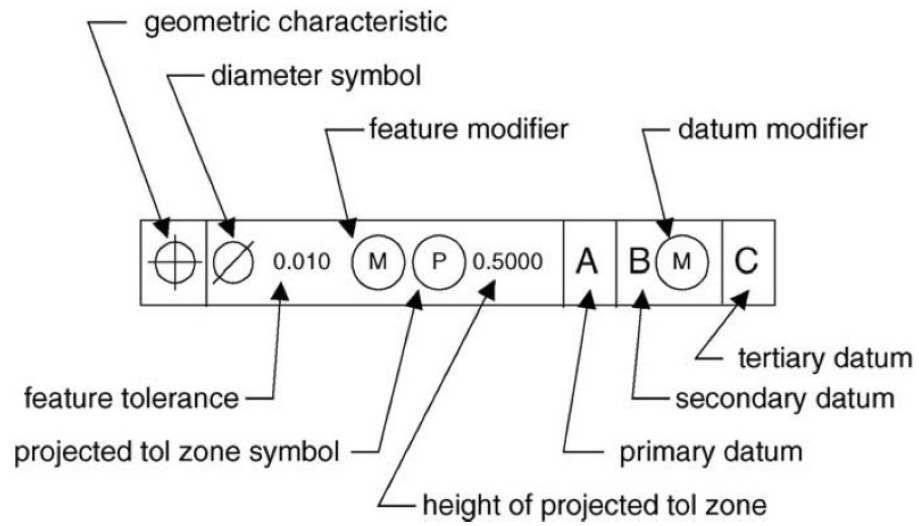

\begin{tabular}{|c|c|}
\hline Term & Symbol \\
\hline diameter & $\varnothing$ \\
\hline spherical dia & $\mathrm{s} \varnothing$ \\
\hline max material cond & M \\
\hline least material cond & ( \\
\hline projected tol zone & P \\
\hline free state & (F \\
\hline tangent plane & T \\
\hline statistical tolerance & ST \\
\hline
\end{tabular}

Figure 1. A typical feature control frame and its possible symbols 
This information is summarized in Table 1 and is quite complete. In this table, Level 0 includes the necessary information for all geometric tolerances. Information of Level 1 and Level 2 often appears in the second compartment of a feature control frame. They are both optional, but Level 1 can be applied to all tolerance types. Information of Level 3 appears in the third compartment of a feature control frame and specifies the datum-related information. Information on the Level 4 appears in the second line of feature control frame under the same geometric tolerance type. Information on the Level 5 is very specific to a certain geometric tolerance and should be incorporated case by case.

\begin{tabular}{lllc}
\hline \multicolumn{2}{l}{ Table 1. Geometric tolerances information summary in ASME Y14.5M-1994 } & \\
\hline Levels & Data elements & Apply to & Required \\
\hline Level 0 & Name & All & Yes \\
& Value & All & Yes \\
& Unit & All & Yes \\
& Applied feature & All & Yes \\
& Tolerance zone type & All & Yes \\
& Label & All & Yes \\
Level 1 & Free state & All & No \\
& Statistical tolerance & All & No \\
& Significant digits & All & No \\
& Description & All & No \\
Level 2 & Feature material condition & Feature with size & No \\
& Projected tolerance zone & Orientation & No \\
& Tangent plane & Orientation & No \\
& Maximum allowable tolerance & Feature at MMC/LMC & No \\
Level 3 & Referenced datum & Orientation, location and runout & No \\
& Datum material condition & Datum feature & No \\
Level 4 & Unit tolerance zone & Flatness and straightness & No \\
& Composite tolerance & Position and profile & No \\
Level 5 & Specific information & Such as all around or partial profile tolerance & No \\
\hline
\end{tabular}

\subsection{DMIS layer}

Though geometric tolerances representation in DMIS is based on ASME Y14.5M-1994, it is specifically modeled for dimensional inspection purpose. Hence, DMIS introduces some new concepts into its tolerance model. A similar approach can be used to summarize geometric tolerances information in DMIS. The information summarized in Table 2 is unique for DMIS, for other geometric tolerances information, refer to Table 1.

In Table 2, Level 0 includes additional necessary information for all geometric tolerances that appears in DMIS. Information of Level 1 is optional and applied to certain types of tolerance. Information on Level 2 is very specific to a certain geometric tolerance that needs to be analyzed and incorporated case by case. 
Table 2. Specific geometric tolerances information summary in DMIS

\begin{tabular}{llll}
\hline Levels & Data elements & Apply to & Required \\
\hline Level 0 & Category & All & Yes \\
& Inspection result indicator & All tolerance actual & Yes \\
Level 1 & Lim & Toleranced feature with size at MMC/LMC & No \\
& Parpln & Orientation tolerance & No \\
& Lotol, uptol & Profile tolerance & No \\
Level 2 & Specific information & Such as AVGDEV value for surface profile tol- & No \\
& & erance actual & \\
\hline
\end{tabular}

\subsection{IMP layer}

In IMP, geometric tolerances modeling should also include information from STEP to make the model more complete. The geometric tolerances modeling in STEP can be found in AIC 519 and some application protocols that need geometric tolerances information, such as AP224. Study of the above materials indicated that the geometric tolerances information summarized in Tables 1 and 2 can cover most of the information from STEP and only few information related to some specific tolerance types is missing. See Table 3 for detailed information.

Table 3. Specific geometric tolerances information summary in STEP AP224

\begin{tabular}{llll}
\hline Level & Data elements & Apply to & Required \\
\hline Level 1 & Unit tolerance zone & Orientation & No \\
& Affected plane & Symmetry, position, parallelism, & No \\
& & perpendicularity, line profile, straightness & \\
& Runout angle & Runout & No \\
& Value qualifier & Position, concentricity & No \\
\hline
\end{tabular}

\subsection{EXPRESS-G model}

Based on the information from Tables 1-3, EXPRESS-G is chosen to abstract the tolerance modeling requirement in IMP. By abstracting data information from above tables into a formal EXPRESS-G representation model, it makes the next step to represent it with XMLrelated technology more convenient.

Figures 2-5 shows a graphical model of layered conformance level for geometric tolerances. In these diagrams, entities with lightest gray conform to the ASME Y14.5M-1994 standard layer (foundation layer); entities with medium gray conform to DMIS 04.0 layer; entities with maximum gray conform to IMP layer. In ASME Y14.5M-1994 layer, we initially create an entity geometric_tolerance based on Level 0 and Level 1 information from Table 1. Subsequently, we classify the entity according to datum information. Finally, we model individual geometric tolerances. 


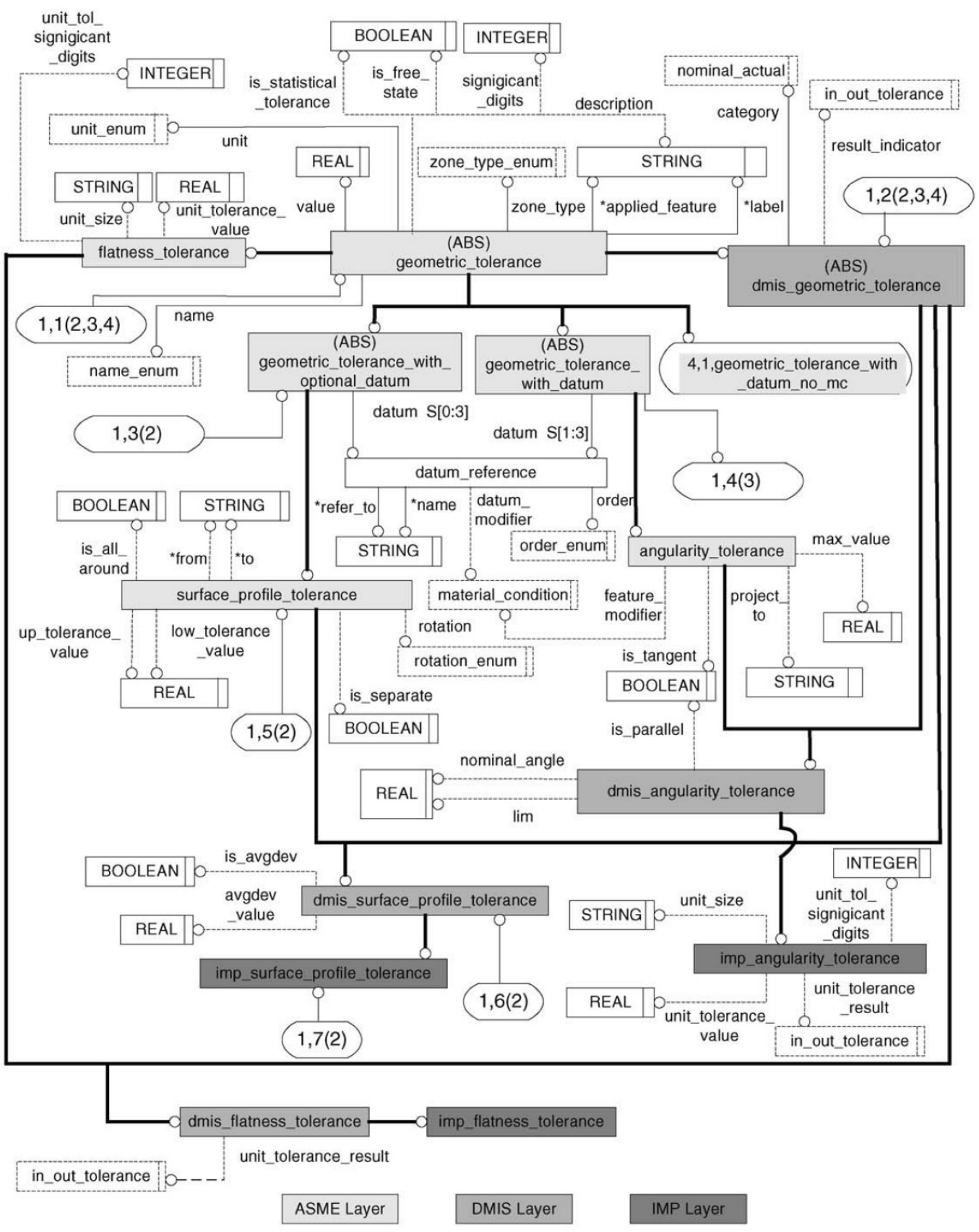

Note : * means a uniqueness constraint is applied

Figure 2. Layered conformance level of geometric tolerances model in IMP (1 of 4) 


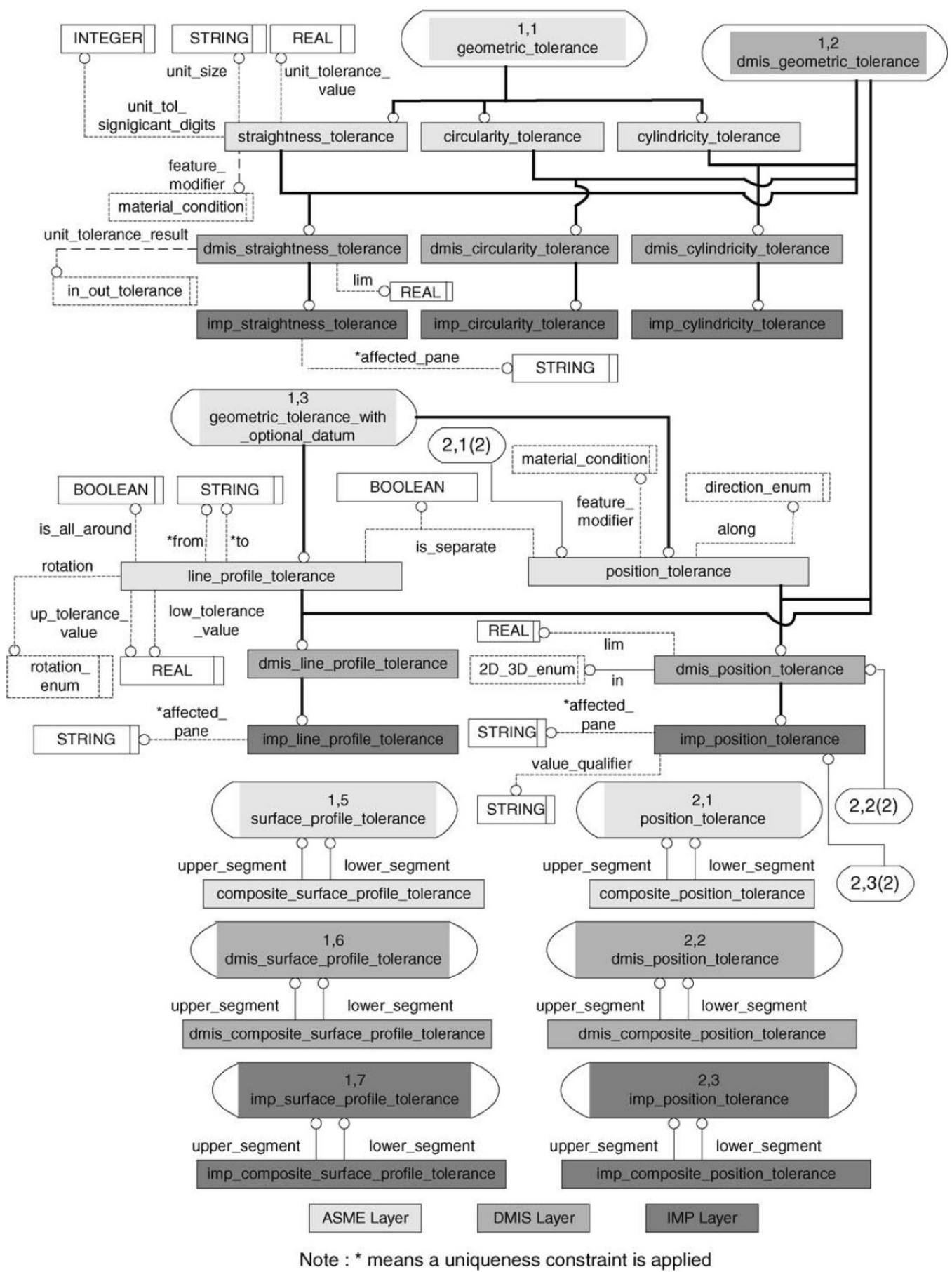

Figure 3. Layered conformance level of geometric tolerances model in IMP (2 of 4) 


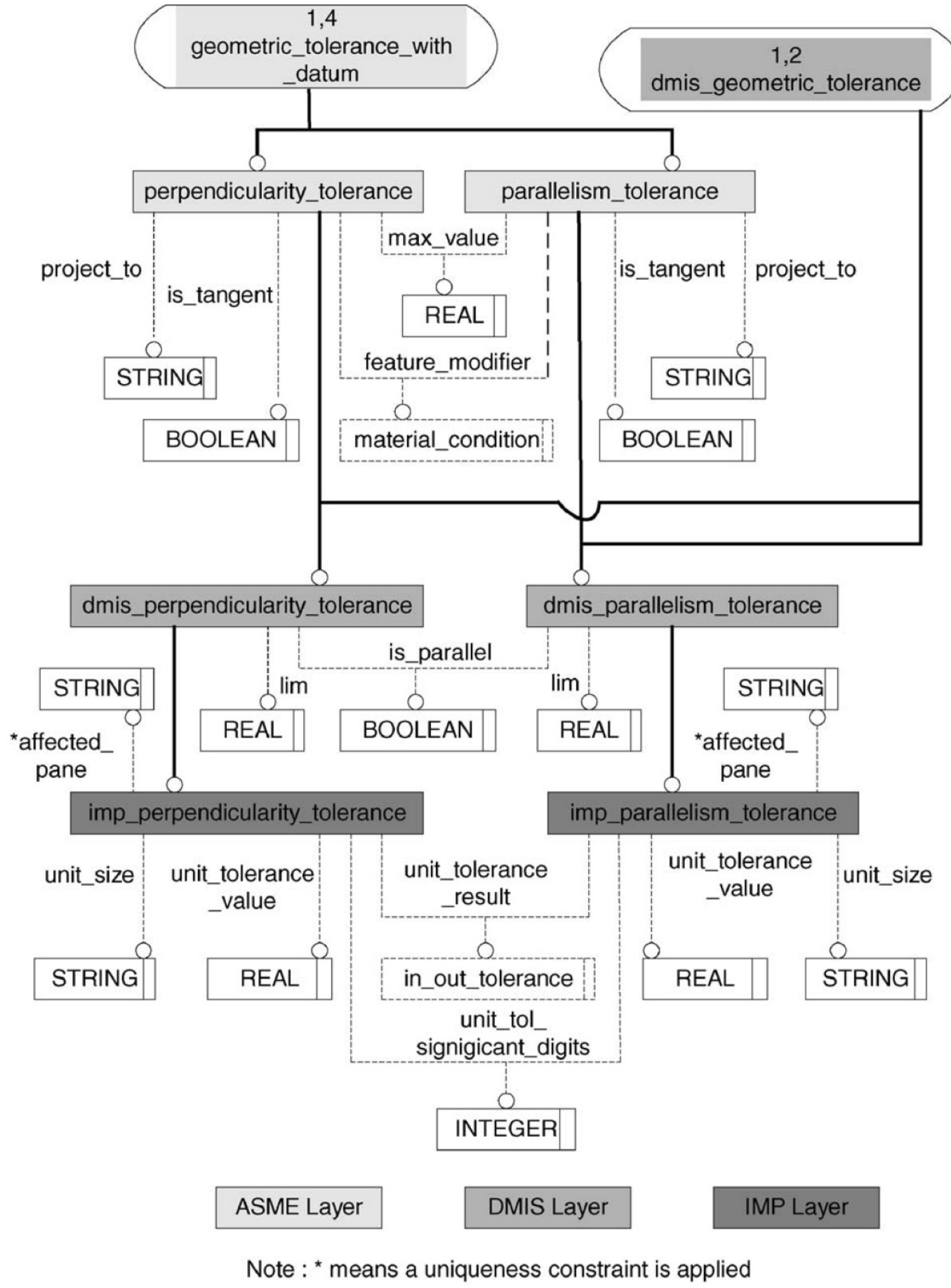

Figure 4. Layered conformance level of geometric tolerances model in IMP (3 of 4) 


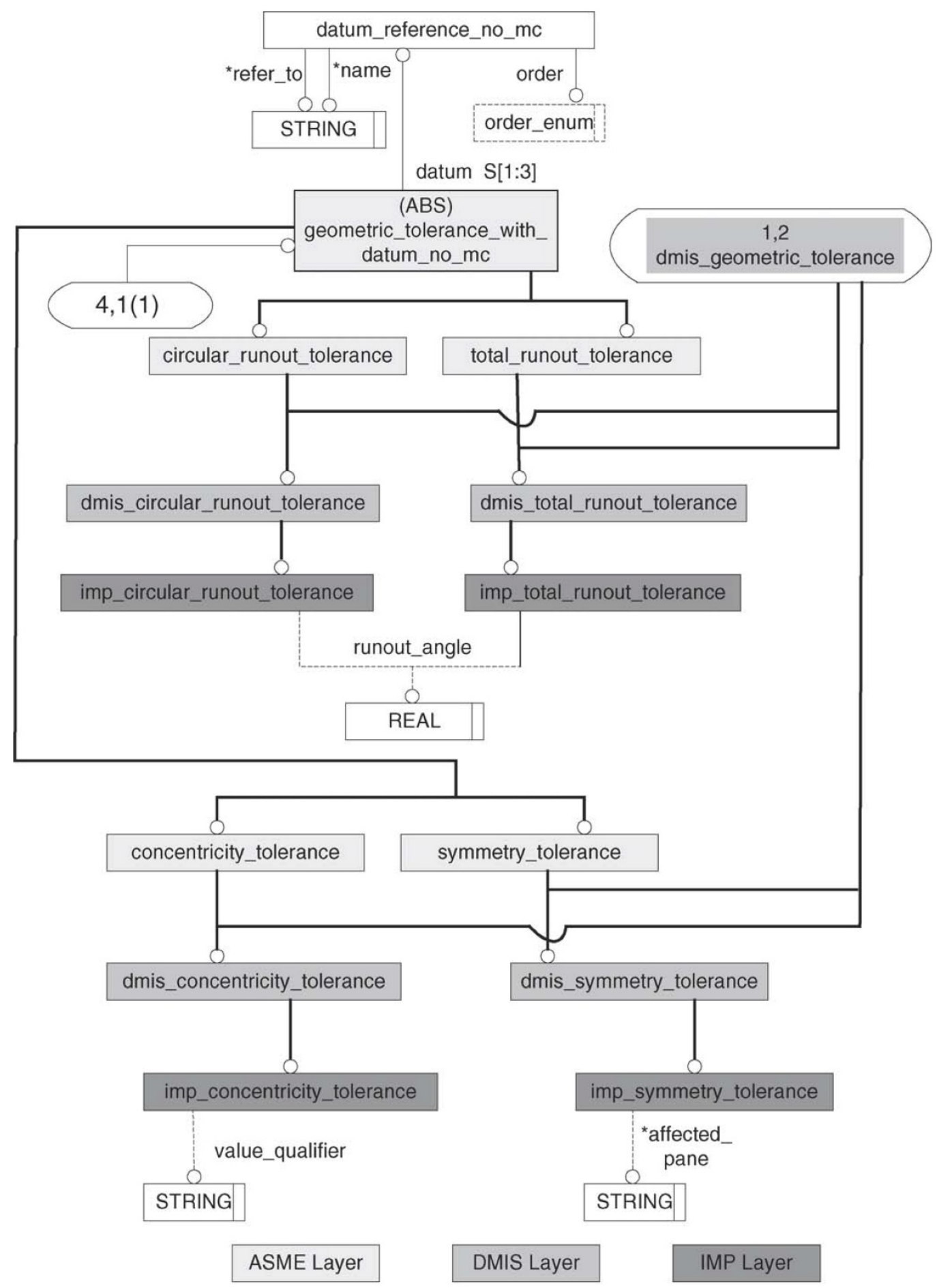

Note : * means a uniqueness constraint is applied

Figure 5. Layered conformance level of geometric tolerances model in IMP (4 of 4) 
Since some geometric tolerances cannot have data, such as flatness, straightness, circularity, and cylindricity; some can have optional data, such as profile of a line, profile of a surface and position; some must have data, such as angularity, perpendicularity, and parallelism; some must have data, but the data cannot have material condition, such as concentricity, symmetry, circular runout and total runout, we further create three entities geometric_tolerance_with_optional_datum, geometric_tolerance_with_datum, and geometric_tolerance_with_datum_no_mc to model these three categories. All of them extend from geometric_tolerance entity and have an attribute datum to describe the datum information but have different lower limit and datum material condition. The definition of other attributes can be traced back to relate standards.

\section{Layered conformance level data representation}

After we model geometric tolerances with EXPRESS-G as shown in Figures 2-5, it is straightforward to create the representation with XML Schema that can be used to instantiate XML file. Since XML Schema does not support multiple inheritances, the information from DMIS is embedded in the lower level entities. Here, we illustrate only how to represent one typical tolerance in EXPRESS_G diagram - angularity with XML Schema. Interested readers can find all types of geometric tolerances representation with XML Schema from [36].

In XML Schema, related elements and datatypes can be grouped together and put in a separate target namespace. These elements and datatypes can be further referred by other target namespaces. By organizing information in this way, it can be easily extended in a hierarchical way. So, before we define the XML Schema file for angularity tolerance, we create a target namespace such as:

$<$ xsd:schema targetNamespace=http://www.coe.uncc.edu/ rgwilhel/geometric_tolerance xmlns=“http://www.coe.uncc.edu/ rgwilhel/geometric_tolerance"

xmlns:xsd="http://www.w3.org/2001/XMLSchema" element FormDefault="qualified" attributeFormDefault="unqualified" version="1.0">

After defining some basic datatypes, we define ASME Y14.5-1994 conformance layer (foundation layer) angularity tolerance datatype based on Figure 2:

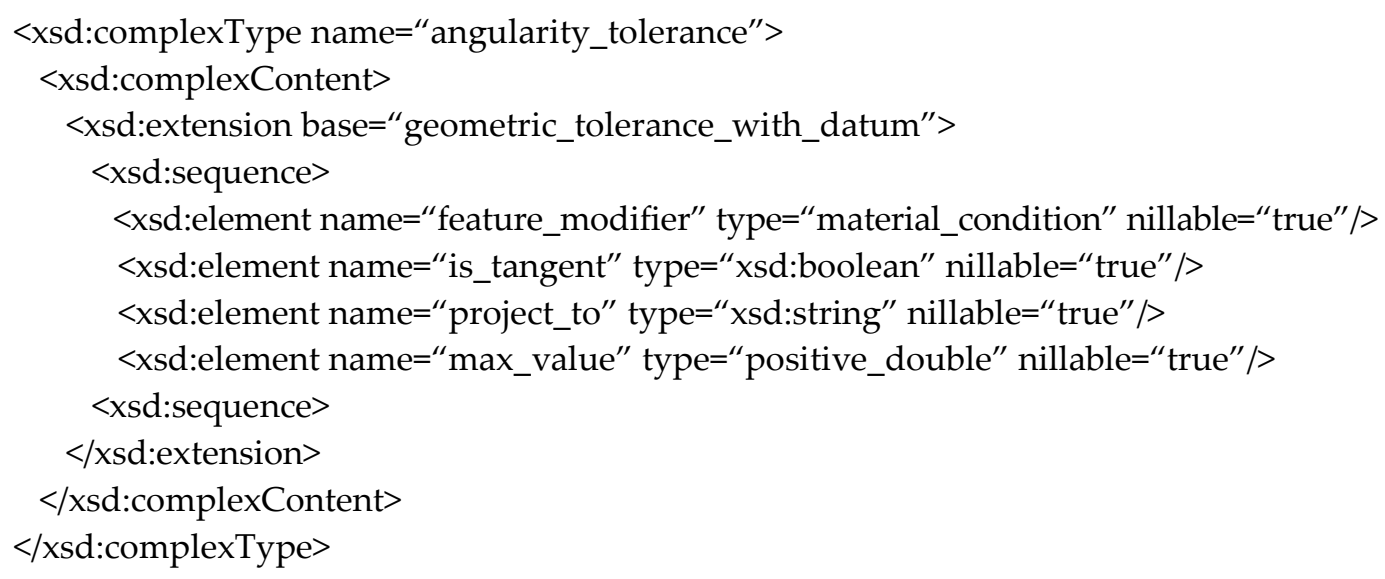


An angularity tolerance conforms to DMIS layer can be defined like:

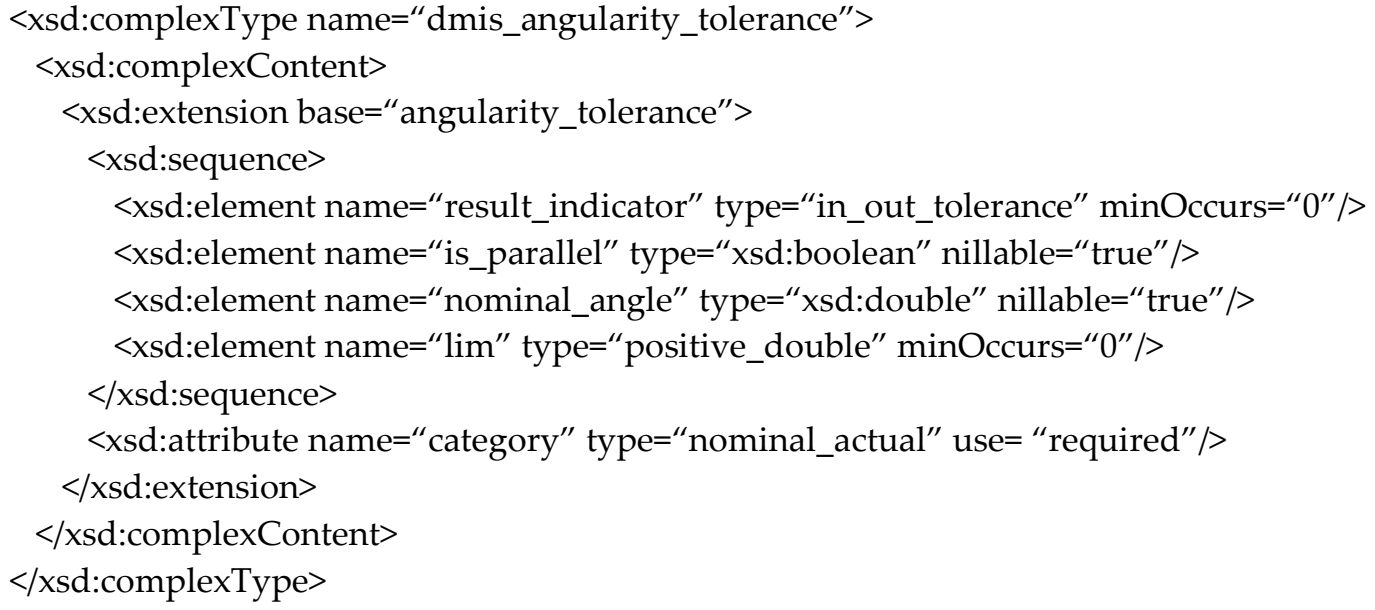

Finally, we define the angularity tolerance that can be used in IMP, like:

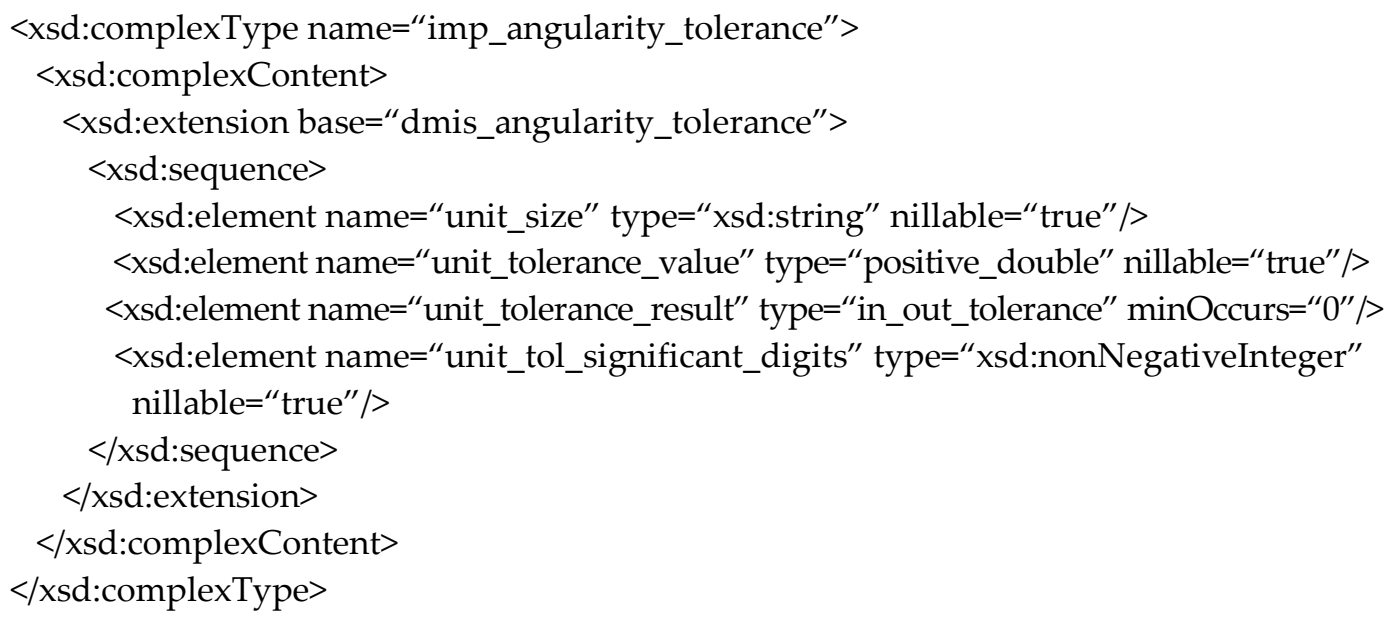

\section{Case study}

After we define these geometric tolerance datatypes in a single XML Schema file, later users can write their own XML Schema file to specify their part's geometric tolerances information by including or importing this file. Finally, they can instantiate an XML file based on their own XML Schema file. This section illustrates this process with a simple example part as shown in Figure 6. This part has a partial unilateral surface profile tolerance applied to the arc, position tolerance for a four-hole pattern, and flatness tolerance applied to the bottom plane. Due to the powerful control ability of surface profile tolerance and its wide application in the auto industry, we use that as an example. The corresponding complete XML Schema file and XML file are available from [36]. 


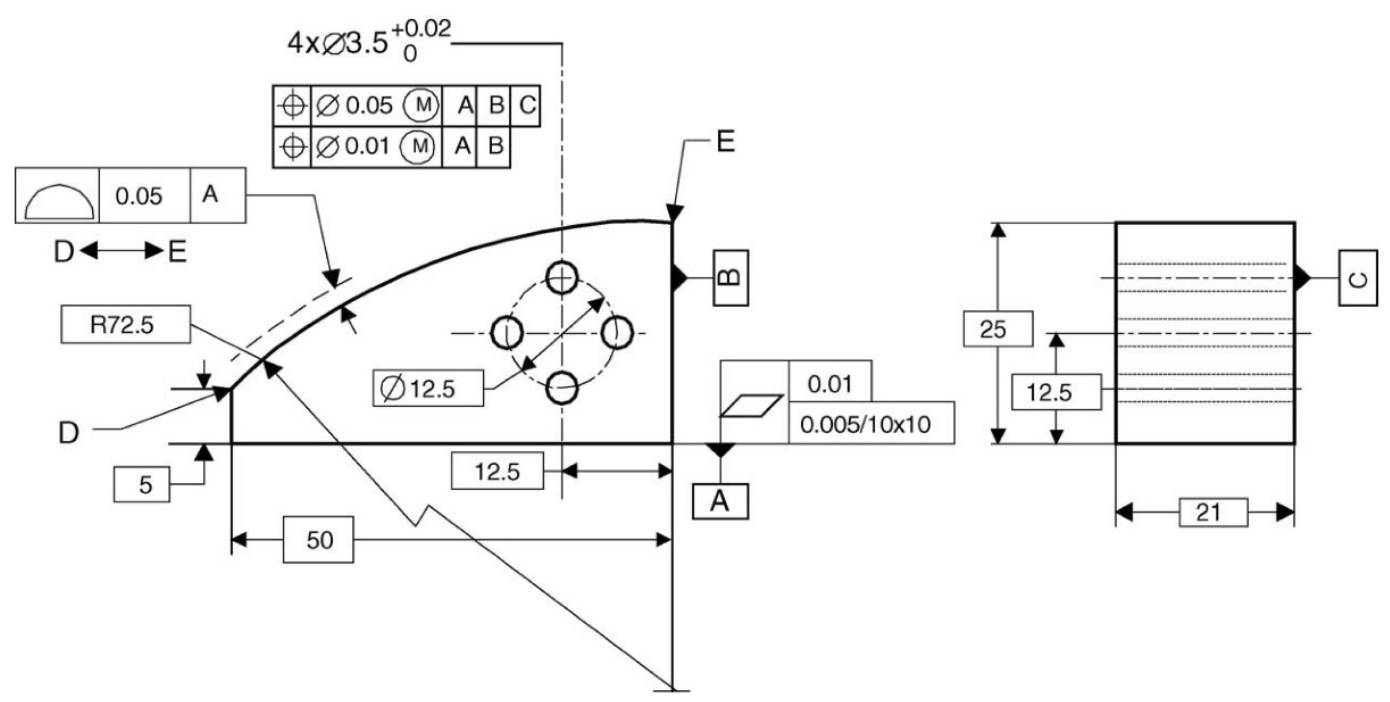

Figure 6. Example part

\subsection{XML Schema file construction}

Here, we use the same target namespace as the geometry_tolerance.xsd. So, it should include predefined geometry_tolerance.xsd.

After defining some auxiliary information, we define the element and datatype for our example part as below:

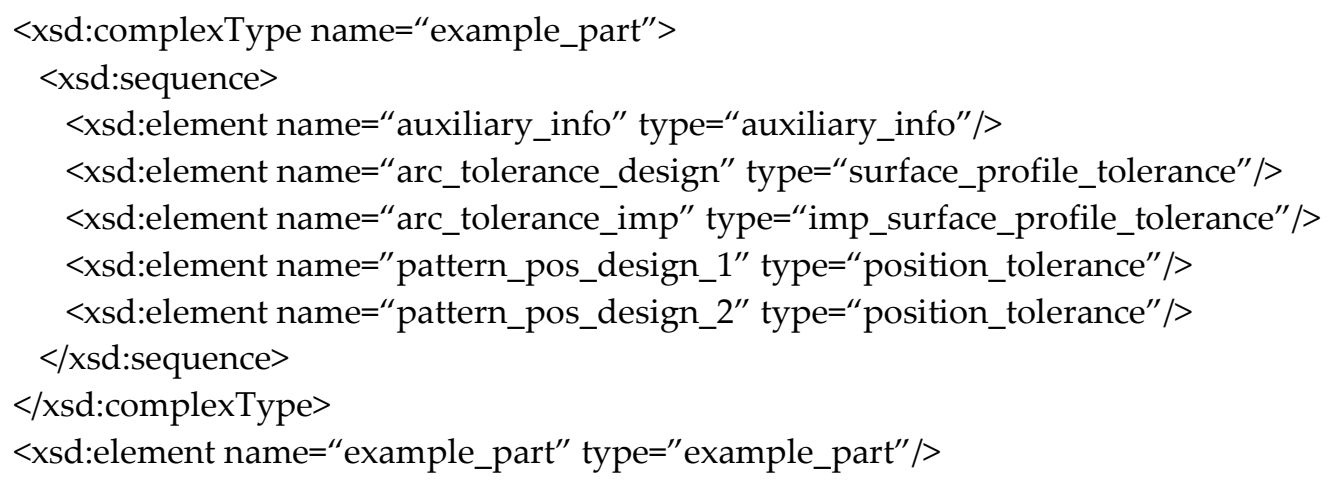

\subsection{XML file instantiation}

Based on the XML Schema file illustrated above, we can construct an XML instance file. In the XML instance file, for its validation, we need to specify the XML Schema file location.

After some dummy features and data are defined, a nominal surface profile tolerance with one datum can be defined at the part design phase like:

<arc_tolerance_design applied_feature="surface_feature_001" label="nominal_sur-

face_profile_001" category="nominal" $>$

$<$ name $>$ surface_profile_tolerance $</$ name $>$

$<$ zone_type $>3 d$ _uniform_boundary $</$ zone_type $>$ 


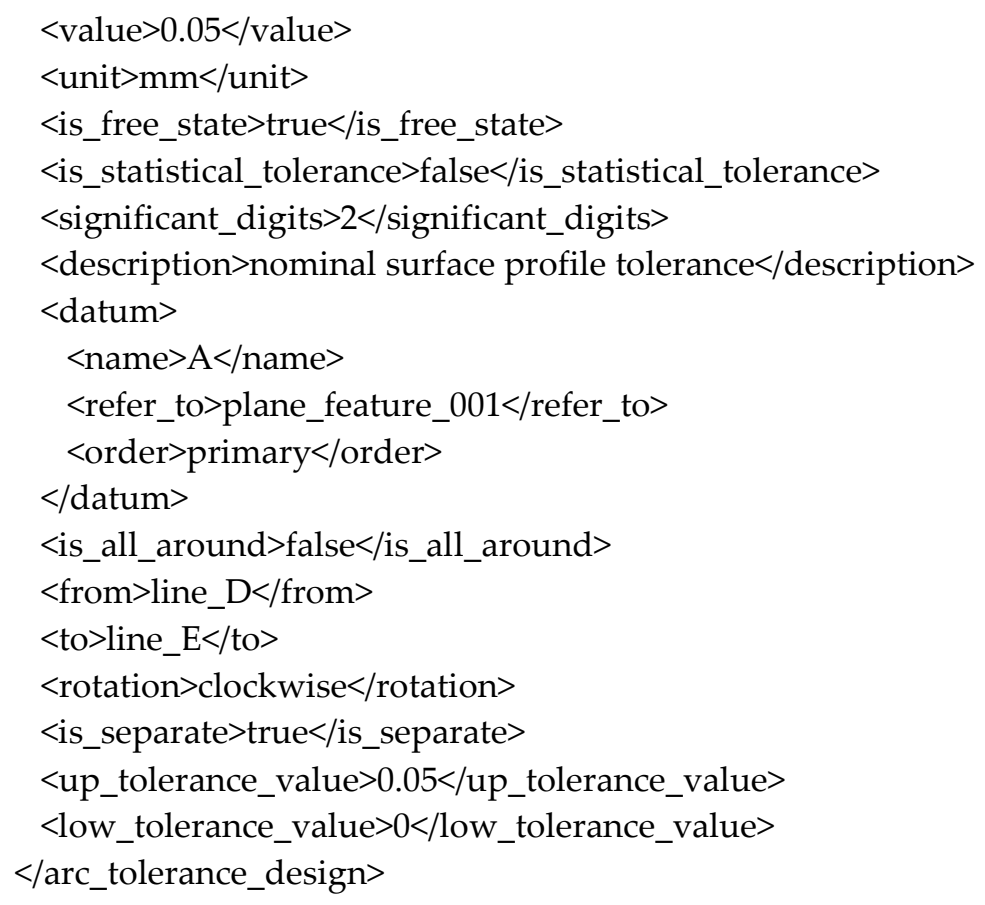

The corresponding actual surface profile tolerance to show inspection result at IMP layer can be instantiated like:

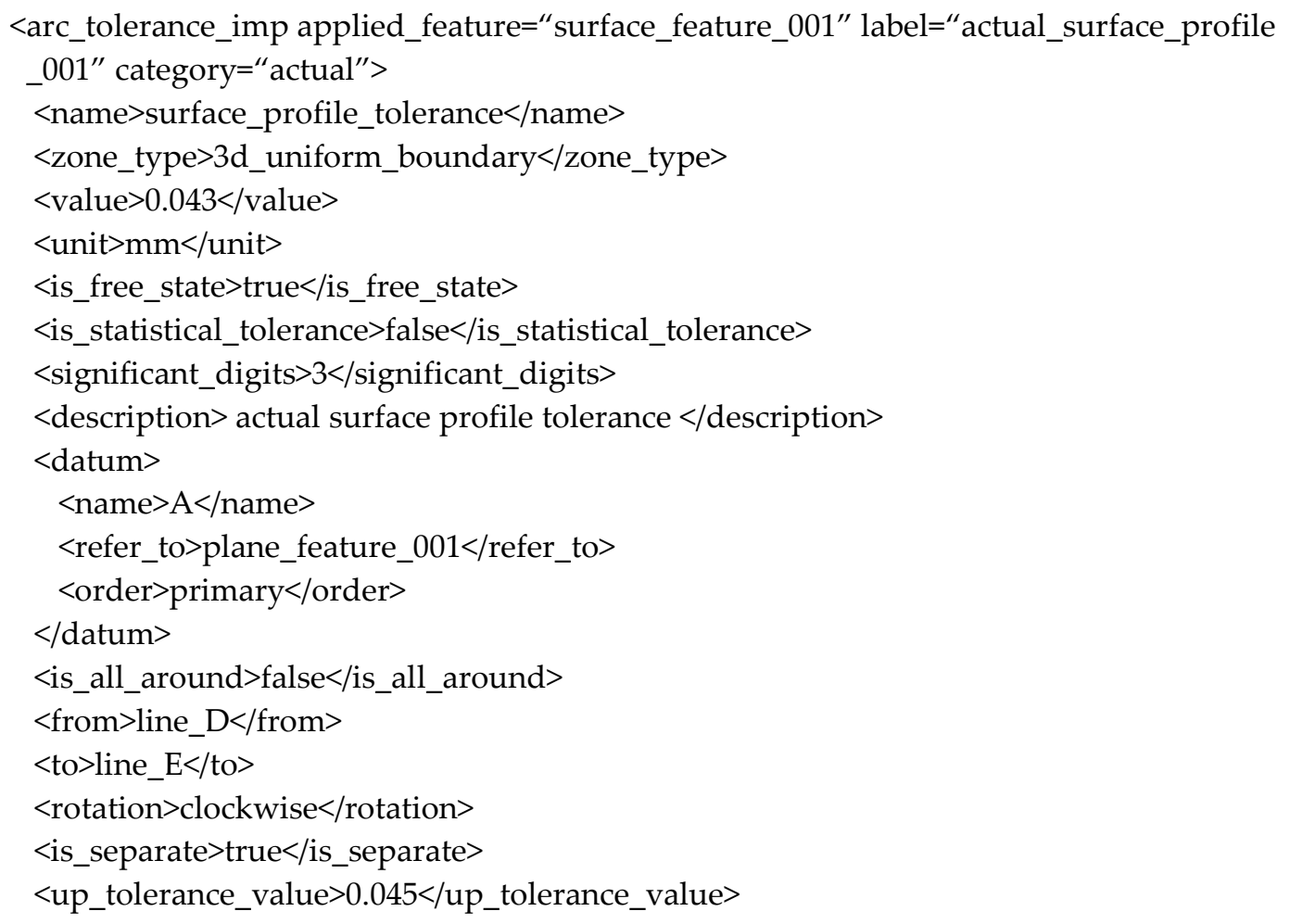




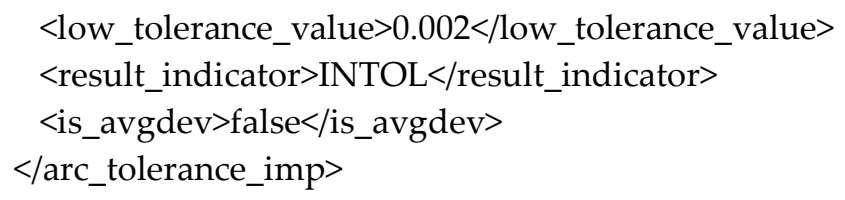

\section{Conclusion}

The requirements of a representation scheme that act as an adapter for the communication of geometric tolerances information is presented. Such a representation is required to meet the needs of the modern manufacturing enterprise for unambiguous communication via the Internet among different application domains. We refer to this communication of geometric tolerances information on a need-to-know basis as Integrated Measurement Processes.

To develop IMP, we present a new layered conformance level data model. This data model has a solid foundation layer by abstracting most geometric tolerances information that appear in the feature control frame and notes from ASME Y14.5-1994 standard. This data model is further extended to form a DMIS layer by incorporating geometric tolerances information from DMIS 04.0 standard and an IMP layer by incorporating geometric tolerances information from STEP standard. Thus, different application domains can take different layer's information to meet their requirements.

XML Schema is chosen to represent our layered conformance level data model since a robust XML instance file needs to have a schema file as its skeleton and XML is a preferred data exchange medium throughout the Internet. Such an XML file is web-applicable, computer-interpretable, and human-understandable. Finally, an example is presented to illustrate the geometric tolerances modeling and representation process of our approach. Additionally, the example provides a brief explanation of the XML Schema and XML instance file construction.

The current development focus of XML and XML Schema at W3C provides data representation and data structure, respectively, to meet data integration requirement, with limited constraints that can be applied to primitives. Thus, they are not appropriate to be used for reasoning and deduction. Neither do they support ontology representation. A separate application is usually required to meet those requirements after parsing data from XML instance file. If $\mathrm{W} 3 \mathrm{C}$ supports these functionalities in the future, the XML schema developed here can be extended.

This work provides a near complete model. However, certain informal information that designers tend to use as notes is not addressed. This model does not attempt to interpret design intent and, similar to the case with actual drawings, interpretation deviations can occur.

Acknowledgments - This work was supported by DaimlerChrysler through the Challenge Fund project entitled "Software Frameworks for Integrated Measurement Processes." We particularly thank Mr. Robert Waite of DaimlerChrysler for his leadership and support. Also, the authors are grateful to ISO 10303-AP 219 and DML committee members for their materials and ideas. 


\section{References}

[1] Y.S. Hong, T.-C. Chang, A comprehensive review of tolerancing research, International Journal of Production Research 40(11) (2002) 2425-2460.

[2] S. C. Feng, A dimensional inspection planning activity model, Journal of Engineering Design and Automation 2(4) (1996) 253-267 (Special issue on Tolerance and Metrology for Precision Manufacturing).

[3] X. Zhao, R. G. Wilhelm, O. A. Martin, J. B. Bullock, Software frameworks for integrated measurement processes, in: Proceedings of the 16th American Society for Precision Engineering, 2001, pp. 273-276.

[4] Metrology Interoperability Project Team, National Institute of Standards and Technology (NIST). URL: http://www.isd.mel.nist.gov/projects/metrology_interoperability/resources.html.

[5] Open Applications Group, Plug and Play Business Software Integration, White Paper, 2000.

[6] A. A. G. Requicha, Toward a theory of geometric tolerancing, International Journal of Robotics 2 (4) (1983) 437-464.

[7] A. A. G. Requicha, Mathematical definitions of tolerance specifications, Manufacturing Review 6(4) (1993) 269-274.

[8] A. A. G. Requicha, S. C. Chan, Representation of geometric features, tolerances, and attributes in solid models based on constructive geometry, IEEE Journal of Robotics and Automation RA2(3) (1986) 156-166.

[9] J. U. Turner, Relative positioning of parts in assembly using mathematical programming, Computer-Aided Design 22(7) (1990) 394-400.

[10] D. C. Gossard, R. P. Zuffante, H. Sakurai, Representing dimensions, tolerances, and features in MACE systems, Computer Graphics and Applications 8(2) (1988) 51-59.

[11] O. Bjorke, Computer-Aided Tolerancing, second ed., ASME Press, New York, 1989.

[12] J.-C. Tsai, M. R. Cutkosky, Representation and reasoning of geometric tolerances in design, Artificial Intelligence for Engineering Design, Analysis and Manufacturing 11(4) (1997) 325-341.

[13] Guilford James, Joshua D Turner, Representational primitives for geometric tolerancing, ComputerAided Design 25(9) (1993) 577-586.

[14] I. Zeid, CAD/CAM, Theory and Practice, McGraw-Hill, New York, 1991.

[15] Shaw C. Feng, Y. Song Eugene, Information modeling of conceptual design integrated with process planning, in: Symposia on Design For Manufacturability, The 2000 International Mechanical Engineering Congress and Exposition, Orlando, Florida, November 5-10, 2000.

[16] ANSI/CAM-I 104.0-2001, Part 1, Revision 04.0. Dimensional Measuring Interface Standard. Computer Aided Manufacturing, International Inc., Arlington, TX, 2001.

[17] ISO 1101:1983(E). Technical drawings, Geometrical tolerancing, Tolerancing of form, orientation, location and run-out-Generalities, definitions, symbols, indications on drawings. International Organization for Standardization, Geneva, Switzerland, 1983.

[18] ASME Y14.5M-1994, Dimensioning and Tolerancing. The American Society of Mechanical Engineers, NY, 1994.

[19] ISO 8015:1985(E). Technical drawings-Fundamental tolerancing principles. International Organization for Standardization, Geneva, Switzerland, 1985.

[20] Industrial Automation Systems and Integration-Product Data Representation and Exchange, Part 1: Overview and Fundamental Principles. ISO 10303-1, ISO, Switzerland, 1994. 
[21] Industrial Automation Systems and Integration-Product Data Representation and Exchange, Part 519: Application Interpreted Constructs: Geometric Tolerances. ISO 10303-519, ISO, Switzerland, 2000.

[22] Industrial Automation Systems and Integration-Product Data Representation and Exchange, Part 224: Mechanical Product Definition for Process Planning Using Machining Features. ISO/TS 10303-224.2, ISO, Switzerland, 2000.

[23] Industrial Automation Systems and Integration - Product Data Representation and Exchange, Part 47: Integrated Generic Resource: Shape Variation Tolerances. ISO10303-47, ISO, Switzerland, 1998.

[24] C. Feng Shaw, Yang Yuhwei, A Dimensional and Tolerance Data Model for Concurrent Design and Systems Integration, The SME Journal of Manufacturing Systems 14(6) (1995) 406-426.

[25] J.-C. Tsai, T.-C. Chung, D.-N. Guo, Development of a STEP-based dimensioning and tolerancing data model, Proceedings of the National Science Council Part A: Physical Science and Engineering 22(6) (1998) 831-840.

[26] Industrial Automation Systems and Integration-Product Data Representation and Exchange, Part 11: The EXPRESS Language Reference Manual. ISO 10303, ISO, Switzerland, 1994.

[27] D. A. Schenck, P. R. Wilson, Information Modeling: The EXPRESS Way, New York, Oxford University Press, 1994.

[28] Industrial Automation Systems and Integration-Product Data Representation and Exchange, Part 219: Dimensional Inspection Information Exchange. Draft-ISO_NWI_ap219_11mar03a .rtf on March 2003; Draft-DIMENSIONAL_INSPECTION.PDF on September 2003.

[29] DML (Dimensional Markup Language), URL: http://www.dmlspec.org/.

[30] M. Rezayat, The enterprise-web portal for life-cycle support, Computer-Aided Design 32(2) (2000) 85-96.

[31] William C. Burkett, Product data markup language: a new paradigm for product data exchange and integration, Computer-Aided Design 33(7) (2001) 489-500.

[32] M. Rezayat, Knowledge-based product development using XML and KCs, Computer-Aided Design 32(5-6) (2000) 299-309.

[33] Industrial Automation Systems and Integration-Product Data Representation and Exchange, Part 28: Implementation methods: XML representations of EXPRESS schemas and data. ISO/TS 10303-28, ISO, Switzerland, 2002.

[34] C. Binstock, D. Peterson, M. Smith, et al., The XML Schema Complete Reference, AddisonWesley, 2003.

[35] Geometric Demensioning and Tolerancing Workbook, The American Society of Mechanical Engineers, Technical Consultants Inc., Florida, 1995.

[36] R. G. Wilhelm, X. Zhao. IMP Data Integration Report. University of North Carolina at Charlotte, 2005. 


\section{Author Biographies}

Xiaoping Zhao is currently a PhD candidate of mechanical engineering and engineering science at the University of North Carolina at Charlotte. He received his BS in mechanical engineering from Beijing Institute of Printing in 1993, his MS degree in mechanical engineering from Xian University of Technology in 1996, and an additional MS degree in computer science from University of North Carolina at Charlotte in 2002. His main research interest is in precision engineering, tolerance analysis, CAD, and system integration.

Trichy Kethara Pasupathy currently works for Hutchinson Technologies, Inc., in the Advanced Process Development group. Prior to this, he worked at the University of Florida, Jacksonville (20032005), Wipro Fluid Power, Bangalore (1994-1998), and Kumaraguru College of Technology, Coimbatore (1990-1991). He received his PhD from University of North Carolina at Charlotte (2003), MS from the Indian Institute of Technology, Madras (1994), and BE from Bharathiyar University, Coimbatore (1990), all in mechanical engineering. His research interests are fluid power, CAD, artificial intelligence, metrology, manufacturing, and magnetic resonance imaging.

Robert Wilhelm is a professor of mechanical engineering and engineering science at the University of North Carolina at Charlotte. As well, he currently serves as the executive director of the Charlotte Research Institute. His research and teaching address computer-aided design, manufacturing, and metrology with a focus on precision engineering. He has industrial experience with Rockwell and Cincinnati Milacron as well as more than two decades of experience in CAD/CAM/CAE. As an internationally recognized expert in precision metrology and mechanical design specification, Wilhelm has directed national and international teams of engineers in the development of mechanical design codes and standards. He currently serves as chair of the Y14.5.1, the standardization effort for mathematical definition of dimensions and tolerances. He has served on the board of directors for the American Society for Precision Engineering and is, by election, a corresponding member of the International Institution for Production Engineering Research. He received his BS in industrial engineering from Wichita State University, his MS degree in industrial engineering from Purdue University, and his PhD in mechanical engineering from the University of Illinois at Urbana-Champaign. In 1994, Dr. Wilhelm received the NSF Young Investigator Award and the SME Outstanding Young Manufacturing Engineer Award. 